In our practice of gastrointestinal endoscopy we have noticed that the use of internal landmarks to determine the anatomical position of the endoscope tip can be inaccurate. This was especially the case during colonoscopy [1]; sometimes it has been required to spray water through the endoscope channel and watch how it fell because this was the only way to confirm the gravitational vertical direction, which does not necessarily match the upper and lower orientation of the video endoscopy monitor.

On this basis, we designed our new device. This is a transparent capsule of the same kind as used in endoscopic mucosal resection [2] but with a double-layered wall. The space between the two walls is almost filled with a transparent liquid, leaving a small air bubble inside. The capsule is attached to the tip of the endoscope (Figure 1), Following the laws of density and gravity, when the tip of the endoscope is rotated in its transverse plane the air bubble will continue to float in its upper position, indicating the vertically upward direction with respect to gravity. When the tip is rotated in its sagittal plane, if the tip is bent downwards,

\title{
A New Device to Clarify the Position of the Endoscope Tip during Endoscopic Diagnosis and Therapeutic Procedures
}

the air bubble will slide up in the opposite direction; if the tip is bent upwards, the air bubble will also slide up in the same direction.

For example when we see on the monitor during colonoscopy that the air bubble is at the right far end of the capsule (Figure 2), this means that the right side of the image is actually the upward vertical direction with respect to gravity in the transverse plane and the tip is bent upwards in the sagittal plane. Comparing these directions with the position of the patient we are able to determine the position of the endoscope tip.

In addition, using the 12 o'clock locating method with this device will help us to determine the exact location of investigated lesions, for later examination or treatment. Finally, based on the same idea, we could use air instead of liquid to fill the space between the two walls of the capsule with a metal or plastic ball instead of the air bubble.

\section{F. Salameh}

Department of Surgery, Tokyo Medical and Dental University, Tokyo, Japan.

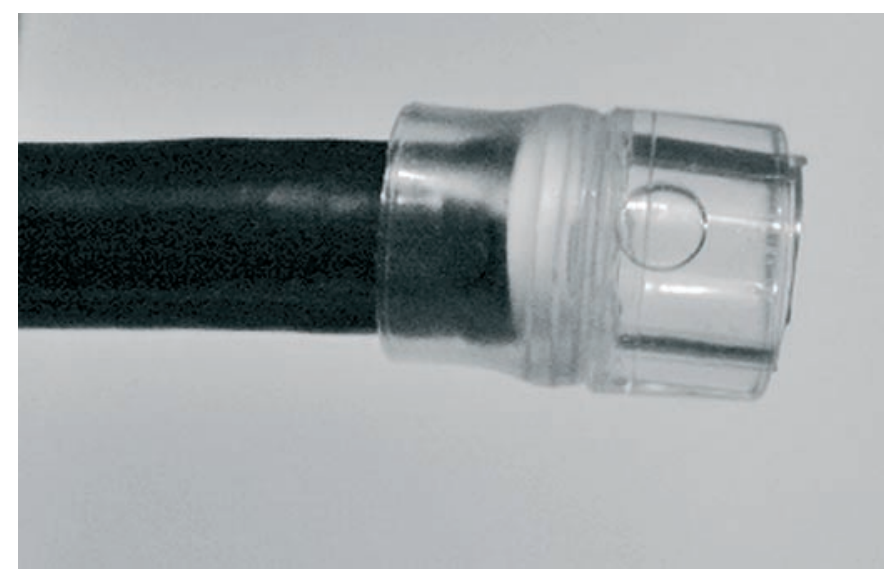

Figure 1 View from above of the doublewalled capsule attached to the endoscope tip, showing the air bubble inside the capsule.

\section{References}

${ }^{1}$ Shah SG, Saunders BP, Brooker JCet al. Magnetic imaging of colonoscopy: an audit of looping, accuracy and ancillary maneuvers. Gastrointest Endosc 2000; 52: $1-8$

${ }^{2}$ Inoue H, Takeshita K, Hori Het al. Endoscopic mucosal resection with a cap-fitted panendoscope for esophageal, stomach, and colon mucosal lesions. Gastrointest Endosc 1993; 39: 58-62

\section{Corresponding Author}

\section{F. Salameh, M.D.}

Department of Surgery 1, Tokyo Medical and Dental University Yushima 1-5-45

Bunkyo-Ku 113-8519

Tokyo

Japan

Fax: $\quad+81-3-38174126$

E-mail: salameh.srg1@tmd.ac.jp

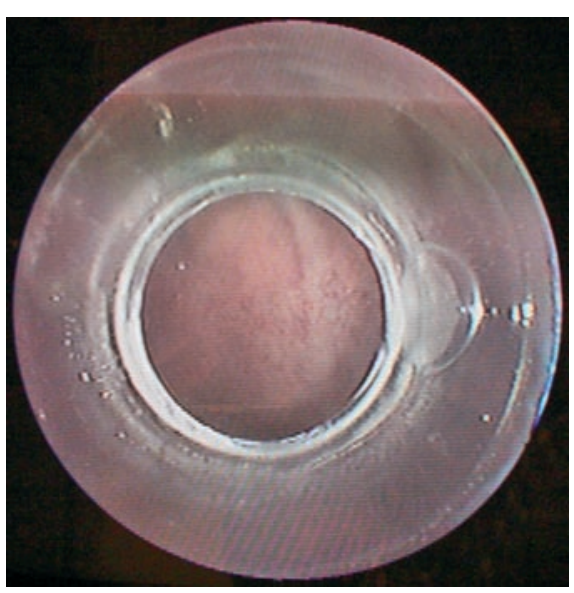

Figure 2 Endoscopic image showing the air bubble at the right far end of the capsule. This indicates that the right side of the image is actually the vertically upwards direction with respect to gravity, in the transverse plane, and the tip is bent upwards in the sagittal plane. 